Meta

Journal des traducteurs

Translators' Journal

\title{
La multiplicité des chemins dénominatifs
}

\section{Judit Freixa Aymerich, Sabela Fernández Silva et M. Teresa Cabré Castellví}

Volume 53, numéro 4, décembre 2008

URI : https://id.erudit.org/iderudit/019644ar

DOI : https://doi.org/10.7202/019644ar

Aller au sommaire du numéro

\section{Éditeur(s)}

Les Presses de l'Université de Montréal

ISSN

0026-0452 (imprimé)

1492-1421 (numérique)

Découvrir la revue

Citer cet article

Aymerich, J. F., Fernández Silva, S. \& Cabré Castellví, M. T. (2008). La multiplicité des chemins dénominatifs. Meta, 53(4), 731-747.

https://doi.org/10.7202/019644ar

\section{Résumé de l'article}

Dans cet article, nous examinons le rôle clé qu'exerce la motivation dans la dénomination en terminologie et nous suggérons que la synonymie présente dans les textes spécialisés peut être expliquée comme le résultat d'une motivation multiple accompagnant l'acte dénominatif. En premier lieu, nous reprenons quelques idées sur la formation des concepts, la dénomination et le rapport concept-terme formulées par les différents courants terminologiques. En second lieu, nous réalisons une analyse sémantique des variantes dénominatives détectées dans un corpus textuel bilingue français-galicien du domaine de la conchyliculture afin de montrer de quelle manière la dénomination ouvre l'accès à la compréhension du concept et comment les différentes variantes dénominatives constituent des points de vue complémentaires sur un même concept. Finalement, nous présentons les possibilités qu'une telle analyse peut offrir pour avancer dans la description et l'explication de la dénomination et de la variation dénominative.
Ce document est protégé par la loi sur le droit d'auteur. L'utilisation des services d’Érudit (y compris la reproduction) est assujettie à sa politique d'utilisation que vous pouvez consulter en ligne.

https://apropos.erudit.org/fr/usagers/politique-dutilisation/ 


\title{
La multiplicité des chemins dénominatifs
}

\author{
JUDIT FREIXA AYMERICH \\ Universitat Pompeu Fabra, Barcelone, Espagne \\ judit.freixa@upf.edu
}

\section{SABELA FERNÁNDEZ SILVA}

Universitat Pompeu Fabra, Barcelone, Espagne sabela.fernandez@upf.edu

\author{
M. TERESA CABRÉ CASTELLVÍ \\ Universitat Pompeu Fabra, Barcelone, Espagne \\ teresa.cabre@upf.edu
}

\begin{abstract}
RÉSUMÉ
Dans cet article, nous examinons le rôle clé qu'exerce la motivation dans la dénomination en terminologie et nous suggérons que la synonymie présente dans les textes spécialisés peut être expliquée comme le résultat d'une motivation multiple accompagnant l'acte dénominatif. En premier lieu, nous reprenons quelques idées sur la formation des concepts, la dénomination et le rapport concept-terme formulées par les différents courants terminologiques. En second lieu, nous réalisons une analyse sémantique des variantes dénominatives détectées dans un corpus textuel bilingue français-galicien du domaine de la conchyliculture afin de montrer de quelle manière la dénomination ouvre l'accès à la compréhension du concept et comment les différentes variantes dénominatives constituent des points de vue complémentaires sur un même concept. Finalement, nous présentons les possibilités qu'une telle analyse peut offrir pour avancer dans la description et l'explication de la dénomination et de la variation dénominative.
\end{abstract}

\begin{abstract}
In this article, we explore the important role that motivation plays in term naming and we suggest that synonymy in specialized texts can be explained as a result of a multiple motivation that takes place during the naming process. Firstly, a short review on the topic of concept formation, naming and the concept-term relationship from different theoretical approaches to terminology is proposed. Secondly, a semantic analysis of the different naming alternatives for the same concept extracted from a bilingual French and Galician corpus on shellfish farming is carried out in order to show how terms are an access door to the understanding of a concept and how the different naming variants are complementary viewpoints on the same concept. We finally suggest in which way such an analysis can contribute to a better description and explanation of the phenomena of naming and synonymy in terminology.
\end{abstract}

\section{MOTS-CLÉS/KEYWORDS}

dénomination terminologique, concept spécialisé, rapport concept-terme, variation dénominative, motivation terminologique

\section{Introduction}

La controverse sur la nature arbitraire ou motivée des signes linguistiques est fort connue depuis que Saussure affirma, dans son ouvrage posthume de 1916, qu'il n'existe aucune motivation entre le signe linguistique et la réalité qu'il représente. 
Des années plus tard, cette affirmation a été nuancée par d'autres auteurs. C'est le cas d'Ullmann (1962), qui a constaté que, malgré le principe de l'arbitraire de Saussure, il existe des signes linguistiques motivés par leur référent, comme les unités onomatopéiques, dont le signifiant est formé sur la base d'une similarité avec un aspect de celui-ci. Certains auteurs ont soutenu ultérieurement que, si l'on considère les signes linguistiques dans leur ensemble, on ne peut pas affirmer que l'arbitrarité soit une caractéristique de leur nature, puisque la totalité des unités formées par des procédés morphologiques ou syntaxiques est basée sur les unités plus simples qui en font partie.

C'est beaucoup plus tard que le principe de motivation du signe a été repris par la linguistique cognitive - dans le cadre des sciences cognitives. Depuis cette approche du langage, la motivation est au cœur de beaucoup d'explications sur les unités des langues, dont les unités métaphoriques constituent l'exemple le plus remarquable. Un grand nombre d'unités s'expliquent alors par des concomitances avec le référent qu'elles représentent.

Dans le domaine de la terminologie, la question de la motivation des termes apparaît à la suite d'études sur les langues menées au sein de la linguistique cognitive. Ainsi, plusieurs recherches ont essayé de démontrer que le choix d'une forme de dénomination n'est pas aléatoire, mais motivée par le sens auquel elle est associée. Sur le plan cognitif, ceci se traduirait en un lien entre la forme et le concept, de telle sorte qu'à partir de cette dernière l'accès au concept serait facilité.

La dénomination des termes, contrairement à celle des mots, peut certainement avoir un fonctionnement sémantique-référentiel spécifique, mais l'acceptation théorique de cette spécificité n'exclut pas le fait que la dénomination fournit de l'information sémantique complémentaire. En effet, en rendant visible un choix de traits sémantiques parmi d'autres, qui font aussi partie du concept, elle nous transmet une vision particulière de ce dernier.

Certains auteurs parlent de motivation (Kocourek 1991) pour expliquer l'origine de la sélection sémantique cristallisée dans la dénomination; d’autres, se situant dans une perspective psycholinguistique, parlent de saillance conceptuelle (Boisson 1996) ou de saillance perceptuelle (Constantin de Chanay 2001); d'autres perçoivent dans ce phénomène le point de départ pour une distinction entre concept et signifié (DikiKidiri 2002); d'autres, enfin, préfèrent parler de point de vue, de perspective ou de vision du concept (Boisson 2001, Cabré 1999). Notamment, dans Cabré (2003), les unités terminologiques se voient attribuées la caractéristique de la polyédricité, notion qui, appliquée aux concepts, peut expliquer certains aspects concernant la diversification de chaque concept en plusieurs sens, chacun d'eux pouvant se projeter dans une dénomination mais se rapportant dans l'ensemble à un même objet de la réalité. Ce phénomène pourrait se conceptualiser de façon polyédrique en une seule unité conceptuelle qui, lorsqu'elle se matérialiserait dans des unités terminologiques concrètes, pourrait donner lieu à des variantes dénominatives diverses.

Dans le présent article, nous nous proposons d'analyser la manière dont les différentes dénominations, pour un même concept, constituent des chemins dénominatifs qui mettent en évidence une vision particulière de celui-ci. Nous suggérons qu'une analyse simultanée des variantes dénominatives peut aider à faire progresser la compréhension de quelques aspects concernant le phénomène de la dénomination en terminologie, ainsi que des mécanismes qui régissent la variation terminologique, et 
qu'elle peut éventuellement contribuer à révéler si sa présence dans le discours de spécialité renferme une utilité cognitive. Pour illustrer notre réflexion, nous nous appuierons sur des exemples extraits d'un corpus de textes spécialisés dans le domaine de la conchyliculture en langues française et galicienne.

Notre position est fondée sur deux thèses principales: en premier lieu, le principe de polyédricité développé par Cabré $(1999 ; 2003)$ et appliqué au concept. Selon ce principe, un concept est le produit de la catégorisation d'un objet ou référent, cette conceptualisation étant unitaire mais pouvant admettre plusieurs angles ou facettes. Sur la base de Cabré (2003), le caractère polyédrique du concept, sur le plan cognitif, peut se traduire, sur le plan linguistique, en un ou plusieurs sens associés à des dénominations différentes. Et c'est justement dans cette variété à l'intérieur de l'unité que réside la polyédricité. Le deuxième élément théorique sur lequel s'appuie cet article est la constatation de Freixa (2002), selon laquelle la variation dénominative peut avoir des conséquences cognitives (pour l'auteure, une variation conceptuelle [Freixa 2002: 368]), de sorte qu'une dénomination peut se visualiser comme une actualisation d'une des facettes d'un concept polyédrique, tel que nous l'avons établi précédemment.

\section{Quelques prémisses théoriques nécessaires dans notre approche}

Avant d'aller plus loin, il nous semble indispensable de présenter une brève synthèse sur ce qui a été écrit en terminologie sur le concept, la dénomination et le rapport existant entre ces deux éléments, afin d'en retenir les apports les plus remarquables pour notre étude. Il faut toutefois signaler que la synthèse théorique que nous proposons autour de ces notions qui ont tant fait couler d'encre au sein de la terminologie sera inévitablement réduite et quelque peu fragmentaire. Notre but n'est que de situer cette problématique dans l'ensemble de la recherche en terminologie et d'isoler les éléments théoriques sur lesquels nous nous appuyons pour proposer notre analyse, que nous appelons chemins dénominatifs en nous inspirant de l'appellation proposée par Petit (2001).

\subsection{Le concept : comment se forme-t-il et comment l'analyse-t-on?}

Les thèses qui se réfèrent à la formation des concepts spécialisés ont évolué de façon parallèle aux réflexions sur le statut linguistique de l'unité terminologique. Dans un premier stade, marqué par la prééminence de la Théorie générale de la terminologie (TGT), la conception logiciste des concepts dans la tradition aristotélicienne prédomine: ils sont conçus comme des unités de connaissance qui organisent la réalité que nous saisissons à travers notre entendement, indépendamment du système linguistique qui les représente. Au fil du temps, la discipline terminologique adopte une orientation plus descriptive, notamment grâce au fait que ce ne sont plus seulement les spécialistes qui s'intéressent à son utilité ou les philosophes à sa nature, mais aussi et particulièrement les linguistes qui apportent de nouvelles perspectives à son étude et explication. Ainsi, cette vision statique du concept est abandonnée en faveur d'une conception plus souple. Une diversité de paramètres jusqu'à alors ignorés sont pris en compte, et la communication spécialisée qui véhicule ces concepts commence à être envisagée non seulement comme un système de représentation des connaissances, 
mais aussi comme le produit d'une interaction entre des agents sociaux, le reflet d'une conceptualisation déterminée ou un système de communication qui intègre toutes ces facettes.

En effet, dans la TGT, Eugen Wüster postule que les concepts existent indépendamment du signe linguistique qui les représente, et qu'ils sont formés dans une phase préalable à celle de l'attribution d'une dénomination. C'est pourquoi les concepts se situent au centre de l'intérêt des terminologues, puisqu'ils constituent ces entités stables qui jouent un rôle clé dans la structuration des connaissances d'un domaine de spécialité quelconque. La délimitation des concepts représentés par les termes (terme ici étant considéré comme équivalent de dénomination) et les relations qui s'établissent entre eux forment la structure de ce domaine de spécialité. La vision wüstérienne implique que le domaine des concepts et celui des dénominations sont indépendants: dans un premier temps, nous avons accès au concept, et par la suite, on lui accorde une dénomination, celle-ci n'étant qu'une représentation du concept.

C'est justement cette conception de l'existence du savoir spécialisé, indépendante de tout système linguistique, qui constitue l'élément distinguant l'objet d'étude de la terminologie de celui de la linguistique. Ainsi, nous constatons quau cours des premières années de développement de la discipline, ce sont des concepts qui sont manipulés, et que ceux-ci sont différenciés de façon explicite de la signification que possèdent les mots:

La terminología considera que el ámbito de los conceptos y el de las denominaciones (= los términos) son independientes. Por esta razón los terminólogos hablan de conceptos, mientras que los lingüistas hablan de contenidos de palabras, refiriéndose a la lengua general. (Wüster 1979: 22)

En raison de la forte influence que la TGT a exercé et de la robustesse de ses affirmations, les idées de Wüster sur le concept ont été élevées au statut de dogme pendant des décennies, jusqu'à l'émergence de nouvelles conceptions. En 1995, par exemple, Sager et Kageura ont présenté une vision du concept comme interaction entre la cognition et l'assignation d'un nom (naming), et ont soutenu que lors du procès cognitif, nous rassemblons les éléments que nous saisissons à travers nos sens en fonction de caractéristiques similaires pour former les concepts et les classes de concepts.

In a simplified manner we could also say that perception is a process which maps extensional items to provisionally established and intensionally defined concepts. (Sager et Kageura 1995: 195)

Le principe qui gouverne le processus cognitif de catégorisation est donc notre désir d'ordonner les stimuli de la réalité en fonction de critères ou de traits que nous considérons saillants et d'en faire des groupes. Ceci nous mène à chercher des traits semblables et à rejeter ceux qui ne le sont pas.

Geeraerts et al. (1994), selon la perspective de la sémantique cognitive et avec une conception du concept spécialisé héritée directement de la théorie du prototype, emploient l'appellation catégories pour référer aux concepts, et affirment qu'il s'agit de catégories mentales non objectives, qu'elles n'existent pas indépendamment de la pensée humaine, et que c'est pour cela qu'elles doivent être étudiées dans leur contexte expérientiel.

Gaudin (2003), qui puise ses idées dans le courant sociolinguistique, défend l'existence d'une interaction entre les concepts et le langage, en même temps qu'il 
met en valeur le rôle actif des agents sociaux dans la construction et l'actualisation continue du signifié dans les productions textuelles. Gaudin reprend les idées de théoriciens comme Putnam, pour qui la langue joue un rôle fondamental dans la construction du savoir. Le monde référentiel que nous percevons à travers notre catégorisation particulière devient façonné par l'instrument du langage. Afin de mieux comprendre le processus de catégorisation de la réalité, il est nécessaire de tenir en compte quatre éléments majeurs: 1) Il existe un rapport entre la langue et la réalité. 2) Référer est un acte ou un procès mis en place par des agents sociaux. Par conséquent, ce phénomène est soumis, d'une part, aux changements imposés par l'échange entre locuteurs et, d'autre part, au passage du temps. Cela explique que le contenu des concepts puisse varier, augmenter ou diminuer et que les dénominations puissent demeurer sans que les ontologies s'en voient forcement altérer. 3) L'acte de référer est interlocutif, dialogué et il est le résultat de l'interaction. 4) Les textes occupent une place majeure dans la construction de la signification et, en même temps, dans la construction de référents.

Les significations sont fixées dans des discours. Et ce sont eux qui les font évoluer, notamment vers plus ou moins de précision dans la construction de la référence. (Gaudin 2003: 42)

D'autre part, la différenciation entre concept et signifié a fait l'objet de débats entre les chercheurs, notamment dès qu'il s'agit d'aborder le travail d'analyse sémantique. Ainsi, pour certains auteurs, le concept est considéré comme un équivalent du signifié. Cabré $(1999$; 2003) résout cette dichotomie entre concept et signifié en les situant sur deux plans différents: concept sur le plan cognitif et signifié sur le plan linguistique, étant entendu que, sur ce dernier plan, le signifié se projette dans des sens différents. Dans le courant lexicographique, des auteurs comme Thoiron (1996), Boisson (2001) ou Béjoint et Thoiron (2001) affirment que le concept est une unité du système conceptuel qui opère à un niveau cognitif, et qui se différencie du niveau sémantique, constitué de traits sémantiques.

Selon une perspective différente, Diki-Kidiri (2002) propose de distinguer concept et signifié. Il y rajoute la notion de percept, qui renvoie à la manière de signifier:

En nous appuyant sur des données tirées de plusieurs langues et cultures tant africaines qu'européennes, nous montrerons que bien que les notions de concept et de signifié puissent toutes les deux évoquer les mêmes produits culturels de l'esprit humain, le concept semble renvoyer à plus d'objectivité et donc à plus d'universalité dans la représentation des choses, tandis que le signifié, lui, paraît plus étroitement dépendant des perceptions particulières à chaque culture.

Or tout comme il peut changer d'une culture à l'autre, le signifié peut changer dans le temps et l'espace d'une même culture. On peut dire que, pour un même objet donné, le concept en est l'idée essentielle, le principe, ou encore l'archétype, tandis que le signifié en est l'angle de vue, un angle qui implique par définition la possibilité d'autres angles de vue susceptibles d'être sélectionnés. C'est pourquoi nous l'appelons percept par analogie à concept. (Diki-Kidiri 2002: 6)

Lorsqu'on travaille sur la sémantique des termes spécialisés, ceux-ci sont souvent représentés comme un ensemble de traits conceptuels. L'identification de ces traits s'effectue moyennant une série de stratégies, la plus efficace étant d'interroger les 
spécialistes. Le contenu du concept est exprimé au moyen d'une définition, dont les limites sont fixées à travers l'explicitation de ses relations avec d'autres concepts.

D'autres auteurs proposent comme stratégie l'observation des termes dans leur comportement discursif, ce qui permet d'obtenir les contextes dans lesquelles les termes sont insérés. Gaudin (2003) est en faveur de cette stratégie textuelle, car il pense que le terme acquiert son sens complet lorsqu'il est en combinaison avec d'autres mots dans un texte. Il affirme également que réduire le contenu d'un terme à sa définition est une erreur, puisque les concepts sont «des développements, des discours, des constructions». La définition d'un terme limitée à l'établissement de ses relations avec des concepts généraux mène à une perte d'information, étant donné que l'information textuelle est omise et que les changements de signification subis par les termes au cours du temps, ainsi que les actualisations de sens qui se produisent lorsqu'ils sont insérés dans un contexte, nous échappent.

Or, la représentation du concept comme un ensemble de traits conceptuels se révèle insatisfaisante pour un grand nombre de chercheurs. C'est justement la critique qu'en fait le courant de la sémantique cognitive, pour qui un bon nombre de catégories peuvent être mieux décrites à partir d'une structure prototypique qui ne correspond pas à la définition classique de traits nécessaires et suffisants, comme l'a démontré Temmerman (2000).

Malgré ces réticences à l'égard des positions structuralistes, tous ceux qui appliquent une analyse sémantique opèrent, dans une certaine mesure, avec des traits. Certains leur confèrent une utilité heuristique pour la représentation du sens d'un terme, bien qu'en soulignant qu'un concept n'est pas la somme de traits conceptuels. Même Geeraerts et al. (1994) effectuent une analyse componentielle des référents, en y reconnaissant son importance heuristique en tant qu'instrument descriptif.

There can be no semantic description without some kind of decompositional analysis: it is hard to conceive of any form of comparative analysis that does not involve breaking down the comparanda into components and characteristics. (Geeraerts et al. 1994: 38)

\subsection{La dénomination et les chemins vers le concept}

Comme nous l'avons mentionné dans l'introduction, la fonction que l'on attribue à la dénomination terminologique est de distinguer le concept par le nom qui lui est propre. Cela signifie que l'on accorde à la dénomination une particularité sémanticoréférentielle que Kleiber, probablement l'auteur qui a le plus contribué à la problématique de la dénomination, a appelé le paradoxe sémantique de la dénomination.

Dans son travail de 2001, Kleiber révise son célèbre article de 1984, «Dénomination et relations dénominatives", qu'il articule en huit points. Nous nous intéressons ici aux trois premiers, qu'il garde dans la révision de 2001 et que nous reproduisons ici:

1. Le terme dénomination renvoie à la relation entre une expression «X» et un élément « $\mathrm{X}$ » de la réalité. Il dénote également l'expression linguistique utilisée pour la relation de dénomination.

2. Contrairement à la désignation, la dénomination est le résultat d'un acte dénominatif préalable, c'est-à-dire que la dénomination est le résultat de l'instauration d'une fixation référentielle préalable, qui peut être le résultat d'un acte de dénomination effectif ou d'une habitude associative.

3. Ladite association référentielle doit être durable. 
Nous constatons que Kleiber, avec le point 3, ne considère pas comme des variantes dénominatives les paraphrases ou les prédications sur un terme. Cependant, d'autres auteurs divergent sur ce point-là. C'est le cas de Petit (2001), qui traite les phénomènes précédents de dénominations tout en distinguant des types différents : il propose, d'un côté, la dénomination statutaire et, de l'autre, la dénomination occurrentielle. De cette façon, l'ambiguïté de l'opposition se maintient dans la pratique, mais les deux types sont considérés comme des dénominations ${ }^{1}$.

Les travaux récents sur la variation dénominative en terminologie montrent que la plupart des concepts se manifestent sur le plan linguistique à travers une multiplicité de dénominations. Ainsi, l'idéal de biunivocité terminologique de la théorie wüsterienne est nié, bien que la particularité sémantico-référentielle demeure indéfinie. Il est vrai que cette multiplicité dénominative pour un même concept répond à une diversité de causes (Freixa 2005; 2006) et elle part d'une évidence incontestable: même si le signe linguistique est arbitraire, les dénominations terminologiques sont majoritairement motivées, et les possibilités de dénomination sont multiples; c'est pour cela que l'on retrouve différentes dénominations pour se rapporter à un même concept sur le plan cognitif.

La discussion à propos du caractère motivé ou conventionnel du langage acquiert un intérêt particulier lorsqu'on se place dans le domaine de la terminologie. Si l'on observe par exemple la Théorie générale de la terminologie, l'association arbitraire entre dénomination et concept est défendue à un niveau théorique, mais l'on formule parallèlement des directives sur le choix des dénominations dans lesquelles certains traits conceptuels sont priorisés par rapport à d'autres. Ainsi, les caractéristiques intrinsèques sont prioritaires par rapport aux extrinsèques, et parmi ces dernières, on préfère les caractéristiques d'usage à celles d'origine (Wüster 1979: 91). Cette motivation dans le choix de la dénomination a seulement lieu dans les unités composées de plus d'un élément verbal, lesquelles constituent «una definición abreviada de este concepto» (Wüster 1979: 86).

D’après Kocourek (1991: 172-173), toute unité lexicale dont la forme évoque un sens global est arbitraire, tandis qu'une unité motivée est celle dont la forme suggère, en plus du sens global, des éléments de contenu qui indiquent la raison pour laquelle la forme est employée pour désigner le sens donné. Le même auteur affirme qu'en terminologie, la majorité des termes sont motivés, et que la prédominance de la motivation est si prononcée qu'elle constitue un caractère essentiel de la formation terminologique, du fait que la forme des termes suggère souvent une partie de leur sens.

D’autres auteurs, comme Lethuillier (1989), parlent d'évocation, qu'il définit comme la façon dont la dénomination pointe vers le concept, phénomène qui ne présuppose pas la négation de l'arbitraire du signe linguistique, mais qui est en relation avec la manière de désigner. Cette proposition repose sur l'idée que la notion est un schéma de description, lequel est comme un squelette enveloppé d'une substance qui en seraient les caractères constitutifs.

Cette idée est dans la ligne des affirmations de Diki-Kidiri présentées au point 2.1. Diki-Kidiri soutient que le choix d'un point de vue (c'est à dire d'une perception, d'où le terme percept qu'il propose) est tributaire d'habitudes, d'analogies et de stratégies d'appréhension fortement conditionnées par la conscience de l'expérience passée, dont nous pouvons trouver les traces dans la mémoire des mots. Ces considérations l'amènent à déclarer: 
Il s'en suivra que le rapport entre le concept et le percept comporte toujours une part de motivation qui suscite et oriente les choix de dénomination. Le signe linguistique ainsi produit ne peut être totalement arbitraire de ce point de vue. Même lorsque le concept est nouveau, le choix de sa dénomination est motivé et conditionne indirectement la formation du signifiant. (Diki-Kidiri 2002: 6-7)

On ne peut pas faire autrement que de reproduire ses mots exacts quant à la distinction entre concept et percept, tant ses propos sont conformes à notre approche:

Le percept n'est donc pas l'équivalent du concept, mais seulement un pointeur vers le concept, un point d'encrage qui permet de saisir globalement le concept, sans avoir à en reconstituer tous les éléments structurels. (Diki-Kidiri 2002: 13)

La motivation de la dénomination pourrait avoir son origine dans ce que Constantin de Chanay (2001) a appelé saillance perceptuelle, et dans la volonté de sélectionner pour la dénomination l'information la plus appropriée pour le destinataire dans un contexte particulier. En revanche, d'autres auteurs soutiennent qu'il est possible que différentes visions d'un concept cohabitent, ce qui aurait comme résultat la multiplicité dénominative. Ainsi, Boisson $(1996 ; 2001)$, qui s'intéresse à la forme qui rend les termes compréhensibles et interprétables, dans ses propres mots «à la manière dont le concept est atteignable à partir de sa dénomination», affirme ce qui suit:

C'est à partir de ce schéma définitionnel brut, dont on postulera l'existence psychologique comme matériau premier de la dénomination disponible pour les diverses langues, que se fabrique la dénomination. Dans celle-ci, il est procédé à des sélections d'éléments en fonction de leur degré d'informativité. Mais, de plus, la dénomination manifeste divers types d'angles de vue sur l'objet, et ce sont ces diverses visions que nous allons maintenant examiner. (Boisson 2001: 147)

Boisson soutient que l'analyse d'une dénomination dans ses éléments de nomination permettrait de saisir une partie de la signification du concept, qui est symbolisée et qui accorde au signifiant sa motivation, son interprétabilité. C'est le contenu que l'on peut lire dans la dénomination. Sa position coïncide avec la proposition de chemins dénominatifs menant au concept que nous lançons dans cet article.

Pour nous, il s'agit de la voie choisie par le locuteur pour exprimer le concept, à savoir le chemin dénominatif par lequel un aspect du contenu sémantique global a été priorisé. Car les termes non seulement servent à dénommer les concepts, mais ils expriment également un sens par eux-mêmes.

En tenant compte de ce que nous venons d'affirmer, un «chemin dénominatif» ne serait pas plus qu'une option de signification ou de matérialisation d'une facette du concept manifestée dans la dénomination d'une unité terminologique, qui deviendrait ainsi une dénomination motivée conceptuellement. C'est la polyédricité du concept qui permet, à partir de l'unité d'un concept, la multiplicité des sens exprimés à travers des dénominations différentes.

\section{Analyse}

Dans la présente section, nous analyserons quelques exemples de variations dénominatives intralinguistique et interlinguistique. Ce faisant, notre objectif est d'observer de quelle façon les différentes dénominations approchent un même concept, dans le sens où nous l'avons décrit dans les pages précédentes. 


\subsection{Provenance des exemples}

Les exemples que nous avons analysés ont été extraits d'un corpus de textes sur la production conchylicole destinée à la consommation humaine. Ce corpus, qui provient d'un travail précédent (Fernández Silva 2006), est formé de textes spécialisés en galicien et en français (voir Annexe 1) repérés sur Internet, émanant d'organismes officiels, publics ou privés.

\subsection{Méthode d'analyse}

Nous avons procédé à un repérage manuel des unités lexicales se rapportant à un même concept. Nous avons sélectionné uniquement les cas où les variantes dénominatives présentaient au moins un changement lexical. Nous avons donc omis les autres variantes (graphiques, orthographiques, morphosyntaxiques, etc.).

L'analyse est basée sur l'observation de la signification rendue par la forme linguistique des unités. Cette description ne cherche pas à être exhaustive, de même que nous n'avons pas non plus voulu inclure tous les traits; c'est pourquoi nous n’avons consulté ni spécialiste ni glossaire terminologique. Notre but est d'analyser l'information sémantique exprimée dans la dénomination, et de cette façon observer quels sont les traits rendus apparents grâce à des éléments de nomination, et quels sont ceux qui sont omis. L'utilité d'une analyse simultanée des différentes variantes dénominatives réside dans la pluralité de visions fournie pour un seul concept, ce qui nous permet de progresser dans la compréhension du phénomène que nous traitons ici: la variation conceptuelle qui se manifeste à travers la variation dénominative dans les textes spécialisés.

\subsection{Analyse des exemples}

Dans les sections suivantes, nous présentons l'analyse d'exemples documentés dans le corpus textuel sur la conchyliculture, regroupés dans le Tableau 1 en fonction des quatre situations de variation conceptuelle que nous avons distinguées: a) galicien et français, des visions différentes du concept; b) variation conceptuelle se manifestant dans la base dénominative; c) variation conceptuelle se manifestant dans l'extension dénominative; d) variation conceptuelle se manifestant dans la base et dans l'extension.

TABLEAU 1

Exemples analysés

\begin{tabular}{|l|}
\hline Galicien et français, des visions différentes du même concept \\
\hline Ex. 1 \\
Galicien: Marisqueo \\
Français: Conchyliculture \\
\hline Ex. 2 \\
Galicien: Inxesta diaria admisible \\
Français: Doses journalières admissibles \\
\hline Variation conceptuelle se manifestant dans la base dénominative \\
\hline Ex. 3 \\
Galicien: sector [subsector, actividade, rama] do marisqueo \\
Français: activité [secteur] conchylicole \\
\hline
\end{tabular}




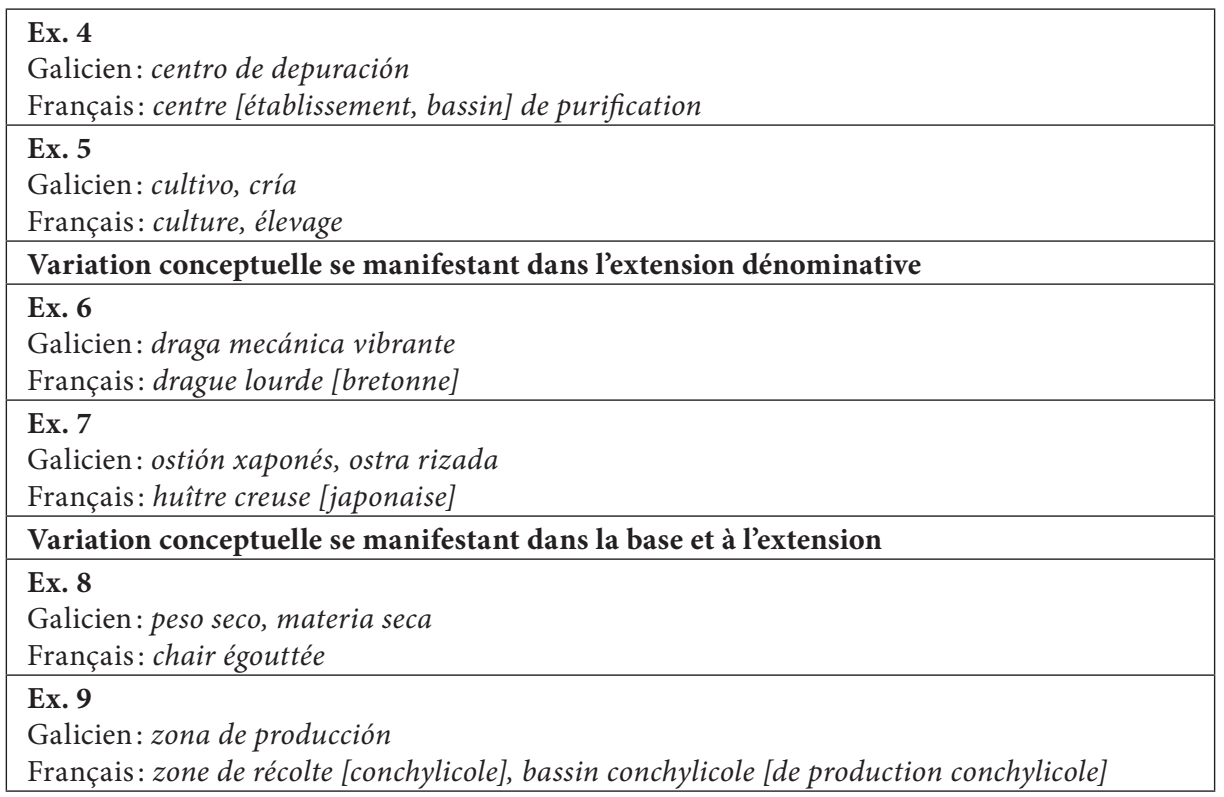

\subsubsection{Le galicien et le français: des visions différentes du même concept}

Nous analysons dans un premier temps les exemples pour lesquels les dénominations ne varient pas à l'intérieur d'une langue ${ }^{2}$, mais qui, d'une langue à l'autre, répondent à des motivations différentes.

a) Marisqueo / conchyliculture

Cet exemple illustre comment un terme monolexémique de base dans le domaine reflète deux visions différentes du concept. La motivation en galicien évoque l'activité humaine désignée par la provenance de la cible de l'activité (la mer), alors qu'en français la même activité est dénommée par le mode d'obtention (culture) et la caractéristique externe de l'objet de l'activité (animaux avec coquille).

b) Inxesta diaria admisible / doses journalières admissibles

Dans cet exemple, nous observons également des perceptions différentes d'un même concept. Ici, les deux langues partagent la même motivation de l'extension (deuxième élément du syntagme terminologique), dans laquelle diaria / journalière renvoie à la période marquée par la durée d'un jour, et admisible / admissible fait référence au fait d'être accepté par l'humain par accord préalable. Par contre, on constate pour chaque langue une motivation différente dans le choix de la base. En effet, pour dénommer une quantité, en galicien nous retrouvons inxesta, c'est-à-dire une mesure établie par rapport à l'action humaine d'ingérer, alors que dans la dénomination française nous retrouvons doses, où l'action mise en valeur est celle d'administrer quelque chose. 


\subsubsection{Variation conceptuelle se manifestant dans la base dénominative}

Dans le cas des unités polylexémiques, les différentes approches du concept s'observent dans les motivations de la base comme de l'extension. En principe, et dans la ligne de pensée que nous avons défendue dans des travaux antérieurs (Freixa 2002), les différentes bases utilisées pour dénommer un même concept ne devraient pas présenter un grand écart sémantique, étant donné que c'est par la base que le concept générique ou superordonné est représenté.

\section{a) Sector [subsector, actividade, rama] do marisqueo}

Sector et subsector ne sont pas, en principe, des unités interchangeables parce que la seconde est rattachée à la première par une relation d'hyponymie. Laissant de côté ces considérations linguistiques, ces unités deviennent des synonymes pragmatiques, car un secteur peut toujours être considéré comme un sous-secteur si un domaine de spécialité est divisé plus finement. Cela n'annule pas la variation existant entre sector (conçu comme une partie différenciée dans l'ensemble des activités de production d'un pays), et subsector (sous-ensemble appartenant à une partie différenciée des activités de production d'un pays), qui est complétée avec les dénominations actividade do marisqueo et rama do marisqueo: dans la première, le concept est présenté comme un ensemble d'activités humaines coordonnées et, dans la deuxième, comme une division dans l'activité humaine de production.

\section{b) Centre [établissement, bassin] de purification}

En français, nous retrouvons également des exemples de variation conceptuelle des bases dénominatives. Ainsi, le lieu où se développe l'action d'éliminer les déchets peut être considéré comme un centre (ce qui met l'accent sur l'effet de concentration ou convergence des activités), un établissement (le lieu ici est désigné par l'action humaine à caractère fonctionnel d'y fixer une activité concrète), ou encore un bassin (où le lieu est défini par sa forme et sa fonction de contenant). Dans ce cas, nous voyons clairement comment chaque terme sélectionne un trait du concept et le rend manifeste dans la dénomination. Bien qu'elles dénomment un seul objet, chacune des dénominations nous en montre une facette différente.

\section{c) Cultivo / culture vs cría / élevage}

Dans cet exemple, les dénominations du français comme du galicien partagent la même alternance conceptuelle; dans ce cas, puisqu'il s'agit d'unités monolexémiques, on ne peut pas parler de variation des bases proprement dites, mais plutôt du lexème qui constitue la totalité de la dénomination.

Dans les deux langues, l'activité humaine que l'on veut nommer apparaît motivée de deux façons différentes, selon que le concept est représenté comme l'activité consistant à traiter le sol en vue de l'obtention de fruits (culture, cultivo), ou comme l'activité qui consiste à nourrir des animaux et maîtriser leur développement en vue de finalités spécifiques (cría, élevage). 


\subsubsection{Variation conceptuelle se manifestant dans l'extension dénominative}

Dans la section précédente, nous faisions allusion au fait que, dans les unités syntagmatiques représentant un même concept, les bases lexicales étaient censées présenter un écart sémantique minimal. L'extension, par contre, correspond généralement au trait qui différencie le concept étudié du reste des concepts subordonnés à un même concept générique, représenté par la base du syntagme. Étant donné qu'un même trait peut être abordé de manières multiples, nous soutenons l'hypothèse que c'est au niveau de l'extension que peut se manifester une variation conceptuelle de plus grande ampleur (Freixa 2002: 231).

\section{a) Draga mecánica vibrante / Drague lourde [bretonne]}

L'unité draga mecánica vibrante (seule dénomination trouvée en langue galicienne) trouve sa motivation dénominative dans deux traits sémantiques différents: le mode de fonctionnement (mecánica) et le mouvement caractéristique (vibrante). Par contre, les deux dénominations françaises documentées dans notre corpus dénomment ce même objet en explicitant deux traits sémantiques différents: une propriété, en l'occurrence son poids (drague lourde), et la provenance géographique de l'objet (drague bretonne).

\section{b) Ostión xaponés et ostra rizada / Huître creuse et huître japonaise}

Mis à part la différence de conceptualisation que l'on constate entre les bases ostión et ostra (ostión faisant référence à une huître de grande taille), nous voulons mettre l'accent sur la variation des extensions du syntagme. Nous observons qu'un trait différent a été privilégié dans le choix de chaque dénomination: soit la provenance (japonaise / xaponés), soit la forme de la coquille (pour le galicien, ondulée [rizada], pour le français, concave [creuse]).

\subsubsection{Variation conceptuelle se manifestant dans la base et l'extension}

Nous allons maintenant analyser les exemples dans lesquels la variation dénominative entraîne une variation conceptuelle se manifestant tant au niveau de la base que de l'extension.

\section{a) Peso seco et ses variantes}

En galicien, nous avons documenté les unités peso seco et materia seca se référant au même concept: dans la première dénomination, la base fait référence à la propriété physique que l'objet possède par l'effet de la gravité, alors que dans la deuxième, on le nomme en fonction de sa nature, en tant que corps présentant un certain volume. Les extensions récupèrent le trait seco, qui informe de son état anhydre.

La forme française chair égouttée est quant à elle le reflet d'une approche dénominative différente: chair fait référence à la composition interne, mais cette fois-ci en tant qu'animal. De même, égouttée nous offre un point de vue différent: celui de l'état résultant d'une extraction de l'eau effectuée par une intervention humaine.

Cet exemple permet de mettre en évidence comment on arrive à une même destination, le concept, par des chemins dénominatifs différents. 


\section{b) Zona de producción et ses variantes}

Pour le terme zona de producción, nous avons documenté les variantes suivantes en français: zone de récolte, zone conchylicole, bassin conchylicole, bassin de production conchylicole.

Nous pouvons constater que les quatre formes françaises présentent des variations dénominatives et conceptuelles en rapport avec les bases et les extensions. Comme nous l'avons vu dans un exemple précédent, zone et bassin évoquent l'objet dénommé sous deux angles différents: le lieu est défini soit comme un espace limité, soit par sa forme et sa fonction de contenant.

En ce qui concerne les extensions, nous retrouvons une alternance entre de récolte (qui souligne l'action effectuée dans l'endroit que l'on nomme, à savoir ramasser sur le sol les objets cultivés), conchylicole (qui privilégie la cible de l'activité, nommée en fonction de la caractéristique externe de posséder une coquille) ou de production conchylicole (dans laquelle l'on constate le choix de rendre explicite deux traits sémantiques: l'action effectuée, cette fois-ci produire, et la cible de ladite action).

\subsection{Commentaire des résultats}

À partir de l'analyse effectuée, nous pouvons extraire quelques comportements généraux concernant le phénomène dénominatif:

1. Pour dénommer des objets, des mesures, des procès, des états, etc. nous pouvons adopter soit le point de vue de l'objet, et faire allusion à une caractéristique qui lui est propre, soit le point de vue de l'humain, et mentionner l'action que celui-ci exerce sur l'objet en question. Ceci explique quelques alternances, comme celle de l'exemple 8 , où matière (materia) et poids (peso) s'opposent à chair. Pour les états, il se produit la même chose avec les variantes seco (objet) ou égouttée (homme).

2. Dans d'autres cas, la variation se produit en fonction d'une caractéristique particulière de l'objet mise en relief par la dénomination:

a) Nous observons diverses manières de dénommer les organismes vivants: par la forme (rizada, creuse), par la provenance (xaponés, japonaise) ou, éventuellement, par d'autres caractéristiques différenciatrices.

b) Pour les objets ou les instruments, nous observons que la dénomination peut mettre en valeur la forme (bassin), une propriété physique (peso, lourde), son mode de fonctionnement (mecánica, vibrante), voire sa provenance (bretonne).

3. Quelques lieux sont dénommés à partir de l'opération que l'on y effectue, celle-ci étant toujours considérée du point de vue de l'humain. Mais ici encore, on constate une variation, selon que l'on mentionne la cible de l'opération (bassin conchylicole) ou l'opération en elle-même (bassin de purification).

4. Les actions proprement humaines présentent des variations en fonction de la caractéristique mise en relief: il peut s'agir de l'action elle-même (récolte), du résultat de l'action (production), de la manière de l'effectuer (culture, cría) ou de la cible de l'action (secteur conchylicole, activité conchylicole, marisqueo).

5. Dans d'autres cas, les différences s'expliquent par la présence de deux langues différentes. En effet, chaque système linguistique est tributaire d'une conceptualisation spécifique associée à la culture d'où il surgit, et cette spécificité se manifeste aussi 
par un mode de dénomination propre. Dans chaque langue, celui-ci est stable, mais les dénominations empruntent des chemins différents dans chacune. C'est le cas des unités marisqueo et conchyliculture. On constate que cette vision se maintient dans toutes les unités syntagmatiques qui incluent cette notion en français (activité conchylicole, bassin conchylicole), et de même pour le galicien (sector do marisqueo, marisqueo, etc.).

\section{Conclusion}

En nous appuyant sur notre analyse, nous sommes en mesure d'affirmer qu'il existe une motivation de la dénomination terminologique, et que la multiplicité de points de vue permettant de représenter un même concept met en évidence que cette motivation n'est pas unique: elle répond, en effet, à une série de facteurs dont la nature nous est encore inconnue. Il paraît certain que chaque dénomination rend manifeste quelques traits et en occulte d'autres, qu'elle souligne une perspective particulière du contenu du concept et que la variation dénominative à base conceptuelle obtenue par la coexistence de plusieurs solutions dénominatives a un effet particulier dans la communication spécialisée. La pluralité de visions qui en résulte pourrait avoir la finalité de faciliter la compréhension du message, contrairement à ce qui a été traditionnellement soutenu à propos de la variation (Wüster 1979: 137). Il est néanmoins probable qu'il s'agisse seulement du reflet linguistique de notre processus de conceptualisation ou de manipulation des catégories mentales.

Quoi qu'il en soit, le présent article nous paraît avoir justifié la pertinence d'une analyse des dénominations visant à explorer la structure des concepts spécialisés, de même que l'utilité d'un traitement simultané de toutes les réalisations linguistiques d'un même concept (variantes dénominatives) de manière à rechercher une réponse aux causes profondes de ce phénomène.

Avec cette proposition des chemins dénominatifs, s'ouvrent de nombreuses voies de recherche sur les mécanismes qui règlent la communication spécialisée. En ce qui concerne le processus dénominatif, un bon nombre de questions nous viennent à l'esprit, brièvement mentionnées ici: existe-il des paramètres qui régissent le choix d'une dénomination, ou la préférence d'une variante dénominative sur une autre? Dans ce cas, quels sont les éléments de la communication qui déterminent ce choix? Le choix serait-il le seul critère, réalisé de façon consciente ou inconsciente, par l'émetteur du texte? Ou peut-être serait-il déterminé par le public, la situation, ou d'autres facteurs sociolinguistiques? Le degré d'informativité des éléments de nomination pourrait-il exercer une influence? Quels sont les traits les plus informatifs pour un concept donné? Dans quelle mesure la dénomination est-elle tributaire du système linguistique dans lequel elle surgit? Quel que soit le chemin choisi, la voie d'analyse des chemins dénominatifs que nous proposons ici nous aidera à résoudre ces interrogations et nous rapprochera sans doute d'une meilleure compréhension du concept spécialisé.

\section{NOTES}

1. Il va sans dire que les formes paraphrastiques et d'autres expressions crées in situ ne seraient pas considérées, de notre point de vue, comme des dénominations. 
2. Nous voulons signaler que cet état de choses concerne seulement l'univers des textes de notre corpus. Cela ne veut pas dire qu'il ne puisse exister des variantes dénominatives pour ces concepts dans d'autres textes.

\section{RÉFÉRENCES}

BÉjoint, H. et P. Thoiron (2001): Le sens en terminologie, Lyon, Presses Universitaires de Lyon.

Borsson, C. P. (1996) : «Les dénominations de la règle à calcul», Meta 41-4, pp. 525-566.

Borsson, C. P. (2001): «Dénomination et "vision” ", Linguistique de la dénomination, Cahiers de praxématique 36 , pp. 141-168.

CABRÉ, M. T. (1999): La terminología: Representación y comunicación. Una teoría de base comunicativa y otros artículos, Barcelona, Institut Universitari de Lingüística Aplicada, Universitat Pompeu Fabra, Sèrie Monografies 3.

CABRÉ, M. T. (2003): "Theories of terminology: their description, prescription and explanation", Terminology 9-2, pp. 163-199.

Constantin de Chanay, H. (2001): «La dénomination: perspective discursive et interactive», Linguistique de la dénomination, Cahiers de praxématique 36, pp. 169-188.

Diki-Kidiri, M. (2002): "La terminologie culturelle, fondement d'une localisation véritable», in Actas del VIII Simposio Iberoamericano de Terminología, Cartagena de Indias, CDROM.

Diki-Kidiri, M. (2000): «Une approche culturelle de la terminologie», Terminologies Nouvelles 21, Terminologie et diversité culturelle, pp. 27-31.

FERnÁndez-Silva, S. (2006): La variación conceptual en terminología: estado de la cuestión y primeras observaciones, Barcelona, Institut Universitari de Lingüística Aplicada, Universitat Pompeu Fabra.

FreIXA, J. (2002): La variació terminològica: anàlisi de la variació denominativa en textos de diferent grau d'especialització de l'àrea de medi ambient, Barcelona, Institut Universitari de Lingüística Aplicada, Universitat Pompeu Fabra, Sèrie Tesis 3.

Freixa, J. (2005): «Variación terminológica: ¿por qué y para qué?», Meta 50-4, CD-ROM.

FreiXA, J. (2006): "Causes of denominative variation in terminology: A typology proposal", Terminology 12-1, pp. 51-78.

GAUDIN, F. (2003) : Socioterminologie. Une approche sociolinguistique de la terminologie, Bruxelles, De Boeck/Duculot.

Geeraerts, D. et al. (1994): The Structure of Lexical Variation. Meaning, Naming and Context, Berlin/New York, Mouton de Gruyter.

KLeiber, G. (1984): «Dénominations et relations dénominatives», Langages 76, pp. 77-94.

KLeIber, G. (2001) : «Remarques sur la dénomination», Linguistique de la dénomination, Cahiers de praxématique 36 , pp. 21-41.

Kocourek, R. (1991): La langue francaise de la science et de la technique, Wiesbaden, Brandstetter.

Lethuiller, J. (1989) : «La synonymie en langue de spécialité», Meta 34-3, pp. 443-449.

Petit, G. (2001): «Pour une conception lexicologique de la dénomination», Linguistique de la dénomination, Cahiers de praxématique 36, pp. 93-115.

SAGER, J.C. and K. KAgEura (1995) : "Concept classes and conceptual structures: Their role and necessity in terminology", Terminology and LSP linguistics. Studies in Specialized Vocabularies and texts. Actes de Langue française et de linguistique 7/8, pp. 191-216.

Saussure, F. de (1916/1976): Cours de linguistique générale, Paris, Payot. [Traduction espagnole (1985): Curso de Lingüística general, Barcelona, Planeta-Agostini]

Temmerman, R. (2000): Towards new Ways of Terminology Description: The SociocognitiveApproach, Amsterdam/Filadelfia, John Benjamins.

Thoiron, P. (1996): «Avant-propos», Meta 41-4, numéro spécial La dénomination, pp. 509511. 
Ullmann, S. (1962): Semantics: An Introduction to the Science of Meaning, Oxford, Blackwell. [Traduction espagnole (1991): Semántica: introducción a la ciencia del significado, Madrid, Taurus].

Wüster, E. (1979): Einführung in die Allgemeine Terminologielehre und Terminologische Lexikographie, Viena, Springer. [Traduction espagnole (1998): Introducción a la teoría general de la terminología y a la lexicografía terminológica, CABRÉ, M. T. (ed.), Barcelona, Institut Universitari de Lingüístic Aplicada, Universitat Pompeu Fabra]

\section{ANNEXE}

\section{Textes qui constituent le corpus}

Auteur/Titre/Localisation

«La charte des bonnes pratiques agricoles et conchylicoles» (Octobre 2001). Section Régionale Conchylicole de Bretage Sud.

$<$ http://www.huitres-de-bretagne.com/PagesConch/CoEnvi01.html>

«Directive du Conseil du 15 juillet 1991 fixant les règles sanitaires régissant la production et la mise sur le marché de mollusques bivalves vivants. (91/492/CEE) ». Journal officiel $n^{\circ}$ L 268, 24/09/1991. (p. 0001 - 0014).

$<$ http://eur-lex.europa.eu/LexUriServ/LexUriServ.do?uri=CELEX:31991L0492:FR: HTML>

«Arrêté du 21 mai 1999 relatif au classement de salubrité et à la surveillance des zones de production et des zones de reparcage des coquillages vivants». Journal Officiel de la République Française no 32, 10/06/1999. (p.08508).

$<$ http://www.cnc-france.com/reglement/>

«Règlement (CE) no 2065/2001 de la Commission du 22 octobre 2001 établissant les modalités d'application du règlement $(\mathrm{CE}) \mathrm{n}^{\circ} 104 / 2000$ du Conseil en ce qui concerne l'information du consommateur dans le secteur des produits de la pêche et de l'aquaculture». Journal officiel $\mathrm{n}^{\circ} \mathrm{L} 278,23 / 10 / 2001$. (p.06-08).

$<$ http://eur-lex.europa.eu/LexUriServ/site/fr/oj/2001/1_278/1_27820011023fr00060008. $\mathrm{pdf}>$

Launay, J. (2001): «La conchyliculture, une activité traditionnelle. La mer et le littoral en Bretagne: pour une ambition régionale». Rennes.

$<\mathrm{http} / / /$ www.bretagne-environnement.org/article1038918317>

IFREMER (2004): «Programme de suivi des effets d'une pollution par des hydrocarbures: propositions de l'Ifremer». [en ligne].

<http://www.ifremer.fr/envlit/surveillance/erikacadrage.htm>

Urien, L.M. (2003) : «La Bretagne, grande région conchylicole». Revue Agreste Bretagne. Direction Régionale de l'Agriculture et de la forêt. Service de statistique agricole. $<$ http:// draf.bretagne.agriculture.gouv.fr/srsa/pdf/4pages/conchyliculture.pdf $>$

LuCAs, R. (coord.) (2004): Chiffres clés de l'environnement en Bretagne. Réseau Bretagne environnement.

$<$ http://www.bretagne-environnement.org/ressources/DOC/pdf/1108137148.pdf>

"Coquillages bivalves filtreurs. Alimentation et croissance». Les nouvelles de l'Ifremer, no21. Le Marin: L'Hebdomadaire de l'économie maritime (Décembre 2000).

$<$ http://www.ifremer.fr/francais/produits/poisson/coquil_biv/marin_coquil_biv_dec2000. pdf $>$

Fifas, S. (2003): «La coquille de Saint Jacques» Les nouvelles de l'Ifremer, n45. Le Marin: L'Hebdomadaire de l'économie maritime (Mars 2003).

$<$ http://www.ifremer.fr/francais/produits/poisson/stjacques/marin_stjacques_stbrieuc_ 2003mars.pdf>

CES GALICIA (2000): «Informe sobre a situación e perspectivas do sector do marisqueo a pé en Galicia». Consello Económico e Social de Galicia. Xunta de Galicia.

$<$ http://www.ces-galicia.org/> 


\section{Auteur/Titre/Localisation}

García Negro, M. C. (2004) : «Consideración sobre o estudo dos danos económicos nos sectores productivos directamente vinculados ás actividades mariñas. O caso do Aegean Sea». Consello da cultura galega, sección de ciencia, técnica e sociedade: Madrid. 354419 .

<http://www.consellodacultura.org/mediateca/pubs.pdf/prestige.pdf>

Nota de prensa da Secretaría Xeral para as Relacións cos Medios Informativos. Xunta de Galicia.

<http://www.xunta.es/periodico/prestige/prestige417.pdf> 